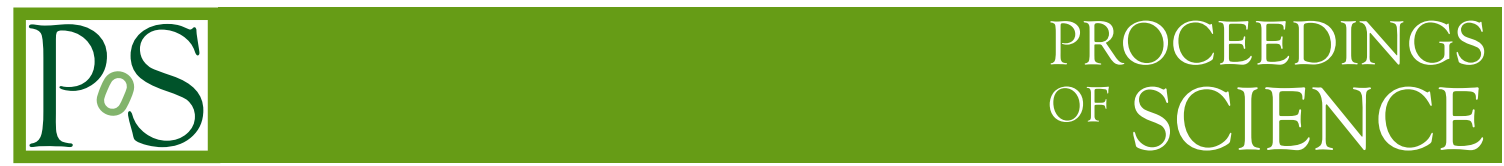

\title{
Exotic and Charmonium(-like) states at BESIII
}

\section{YUTIE LIANG*}

ON BEHALF OF THE BESIII COLLABORATION

II. Physikalisches Institut, Justus Liebig Universität Giessen, D-35392 Giessen, Germany

E-mail: yutie.liang@physik.uni-giessen.de

Recent progress on the exotic and charmonium-like states from BESIII experiment is presented, including the observation of the $1^{3} D_{2}$ candidate $X(3823)$, the observation of $X(3872)$ from $Y(4260)$ radiative decay, and the observation of $Z_{c}$ states.

The European Physical Society Conference on High Energy Physics 22-29 July 2015

Vienna, Austria

${ }^{*}$ Speaker. 


\section{Introduction}

In quark model [1], conventional hadrons are divided into two categories, the quark-antiquark mesons and three-quark baryons. However, Quantum Chromodynamics (QCD) allows the existence of states beyond the conventional hadron, referred as exotic states, such as glueball, hybrids, multi-quark states (states consist of more than 3 quarks). The study of exotic states is important to our understanding of QCD. Despite extensive experimental searches, no unambiguous candidates for any of these exotic configurations have yet to be identified. On the other hand, a number of new particles in the charmonium mass region are observed and show different features from normal charmonium states, and might be good candidates for exotic states.

The BESIII experiment operating at the Beijing Electron Positron Collider II (BEPCII) started to take data since 2009. Since the end of 2012, the experiment has accumulated about $5 \mathrm{fb}^{-1}$ data samples with center of mass energy above $4 \mathrm{GeV}$ and yields fruitful results on the (exotic) charmonium spectroscopy. In this paper, recent progress on the exotic and charmonium(-like) states will be presented.

\section{The $\mathrm{X}$ states}

\subsection{The observation of $X(3823)$}

Recently, the Belle Collaboration reported an evidence for a narrow resonance $X(3823) \rightarrow$ $\gamma \chi_{c 1}$ in B meson decays with a significance level of $3.8 \sigma$ and mass of $(3823.1 \pm 1.8 \pm 0.7) \mathrm{MeV} / \mathrm{c}^{2}$ [2]. This resonance is considered as a good candidate for the $1^{3} D_{2}$ charmonium state.

In BESIII, a search on this $X(3823)$ resonance is performed using the process of $e^{+} e^{-} \rightarrow$ $\pi^{+} \pi^{-} \gamma \chi_{c 1}$ with $4.67 \mathrm{fb}^{-1}$ data above $4 \mathrm{GeV}$. The $X(3823)$ state is observed in the $\pi^{+} \pi^{-}$recoil mass spectra with a statistical significance of $6.2 \sigma$, as shown in the left plot of Fig. 1. The mass of the $X(3823)$ state is determined to be $(3821.7 \pm 1.3 \pm 0.7) \mathrm{MeV} / \mathrm{c}^{2}$, and the width is less than 16 $\mathrm{MeV}$ at a $90 \%$ confidence level. The energy-dependent cross section of $e^{+} e^{-} \rightarrow \pi^{+} \pi^{-} X(3823)$ is shown in Fig. 1 (Right). A fit with the $Y(4360)$ or $Y(4415)$ shape is performed, and both resonance shapes can describe the cross section line shape [3].
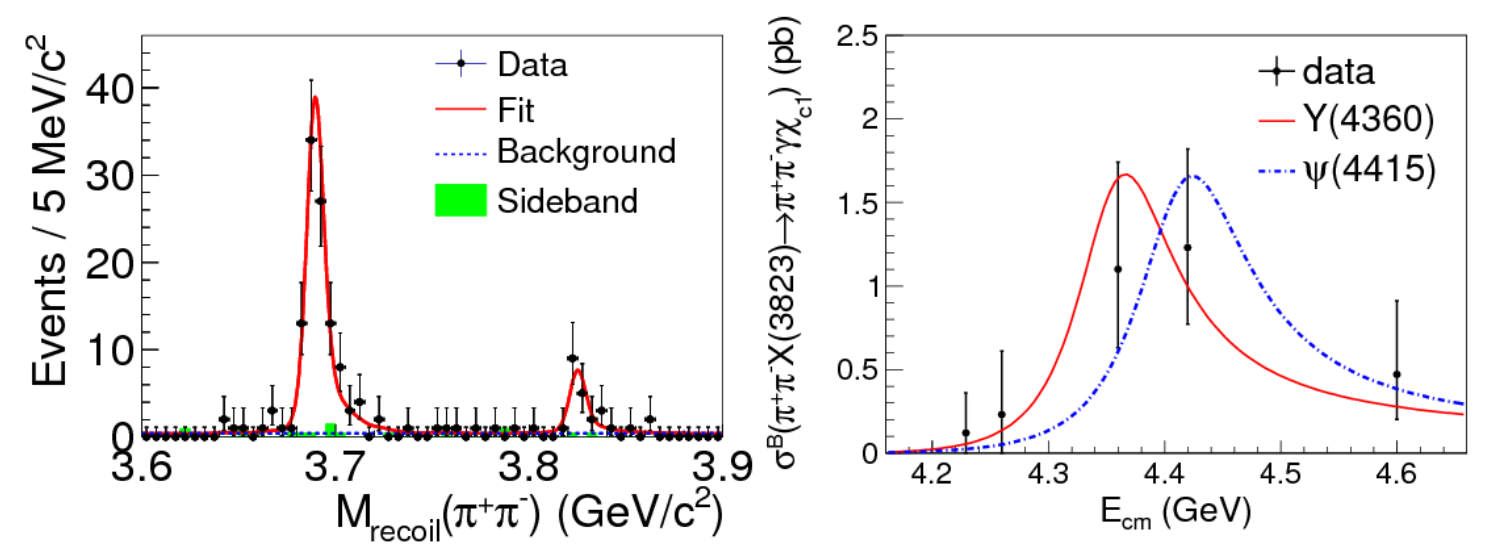

Figure 1: (Left) Fit to the $\pi^{+} \pi^{-}$recoil mass distribution of $\gamma \chi_{c 1}$ events. (Right) Fit to the energy-dependent cross section with the $Y(4360)$ (red solid curve) and the $\psi(4415)$ (blue dashed curve) line shape. 


\subsection{The observation of $e^{+} e^{-} \rightarrow \gamma X(3872)$ at BESIII}

In 2003, the $X(3872)$ state was first observed by Belle in B decays [4]. It was subsequently confirmed by several experiments. Since its mass is close to the $D D^{*}$ threshold, it has been interpreted as a candidate for a hadronic molecule or a tetraquark state.

In BESIII, using $3.3 \mathrm{fb}^{-1}$ data samples at center-of-mass energies from 4.009 to $4.420 \mathrm{GeV}$, the $X(3872)$ is observed in the process of $e^{+} e^{-} \rightarrow \gamma X$ (3872) with a significance of $6.3 \sigma$, as shown in Fig. 2 (Left). The measured mass, $(3871.9 \pm 0.7 \pm 0.2) \mathrm{MeV} / \mathrm{c}^{2}$ is consistent with the previous measurements. In addition, the energy-dependent production cross section of $e^{+} e^{-} \rightarrow \gamma X$ (3872) was measured, and shown in Fig. 2 (Right). It strongly support the existence of the radiative transition process $Y(4260) \rightarrow \gamma X(3872)$ [5].
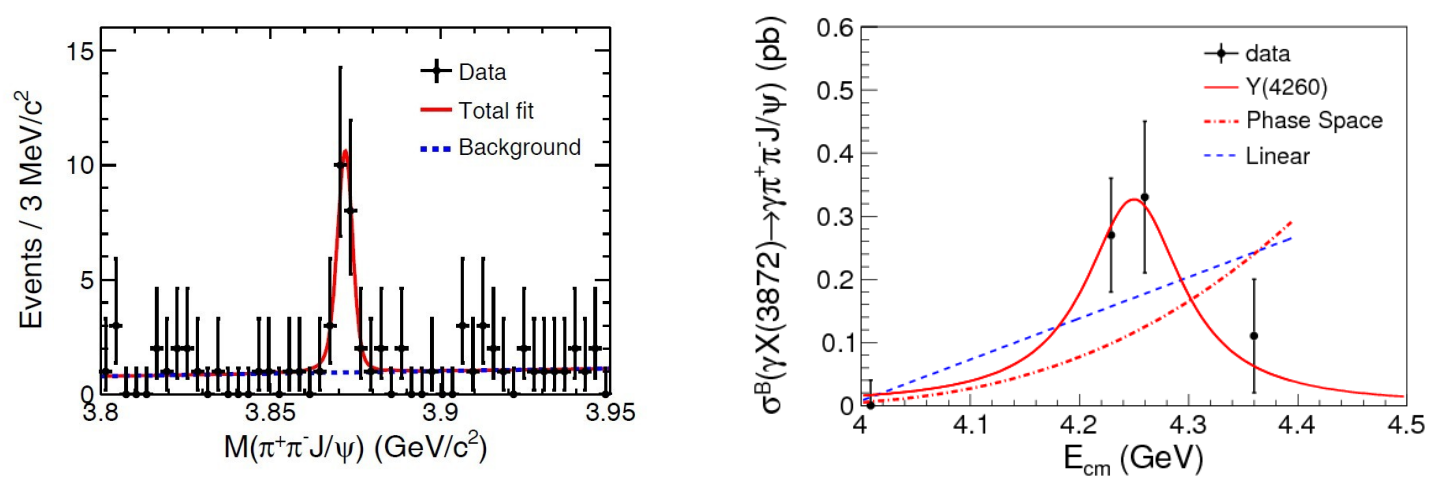

Figure 2: (Left) Fit to the invariant mass of $\pi^{+} \pi^{-} J / \psi$ for $X(3872)$ signal. (Right) Fit to the energydependent cross section with the $Y(4260)$ resonance (red solid curve) and a linear continuum (blue dashed curve) line shape and a E1-transition phase space term (red dotted-dashed curve).

\section{Observations of the $Z c$ states}

\subsection{Observations of $Z_{c}(3900)$ and $Z_{c}(3885)$}

In 2013, BESIII discovered a charged charmonium-like state $Z_{c}(3900)$ decaying into $\pi^{ \pm} J / \psi$ by studying the process $e^{+} e^{-} \rightarrow \pi^{+} \pi^{-} J / \psi$ with $525 \mathrm{pb}^{-1}$ data sample at $4.26 \mathrm{GeV}$ [6]. This state can be seen in Fig. 3 (Left). The mass and width of this state are determined to be $(3899.0 \pm 3.6 \pm$ 4.9) $\mathrm{MeV} / \mathrm{c}^{2}$ and $(46 \pm 10 \pm 20) \mathrm{MeV} / \mathrm{c}^{2}$, respectively. This state was observed by Belle [7] almost at the same time, and shortly confirmed by a paper using the CLEO-c data [8]. This state can not be a conventional charmonium state, since it carries electric charge. The minimal quark content is a four quark combination.

To reveal the nature of the $Z_{c}(3900)^{ \pm}$state, its neutral partner $Z_{c}(3900)^{0}$ has been searched in the process $e^{+} e^{-} \rightarrow \pi^{0} \pi^{0} J / \psi$ using $2.8 f b^{-1}$ data samples from 4.19 to $4.42 \mathrm{GeV} . Z_{c}(3900)^{0}$ is found with a statistical significance of $10.4 \sigma$, as shown in Fig. 3 (Right). The mass and width are determined to be $(3894.8 \pm 2.3 \pm 2.6) \mathrm{MeV} / \mathrm{c}^{2}$ and $(29.6 \pm 8.2 \pm 7.3) \mathrm{MeV} / \mathrm{c}^{2}$, respectively. The discovery of $Z_{c}(3900)^{0}$ indicates the isospin triplet state $Z_{c}(3900)$ has been established [9].

The mass of $Z_{c}(3900)$ is close to the $D \bar{D}^{*}$ threshold, which is suggestive of a virtual $D \bar{D}^{*}$ moleculelike structure. The BESIII experiment did a study on the process of $e^{+} e^{-} \rightarrow \pi^{ \pm}\left(D \bar{D}^{*}\right)^{\mp}$ 

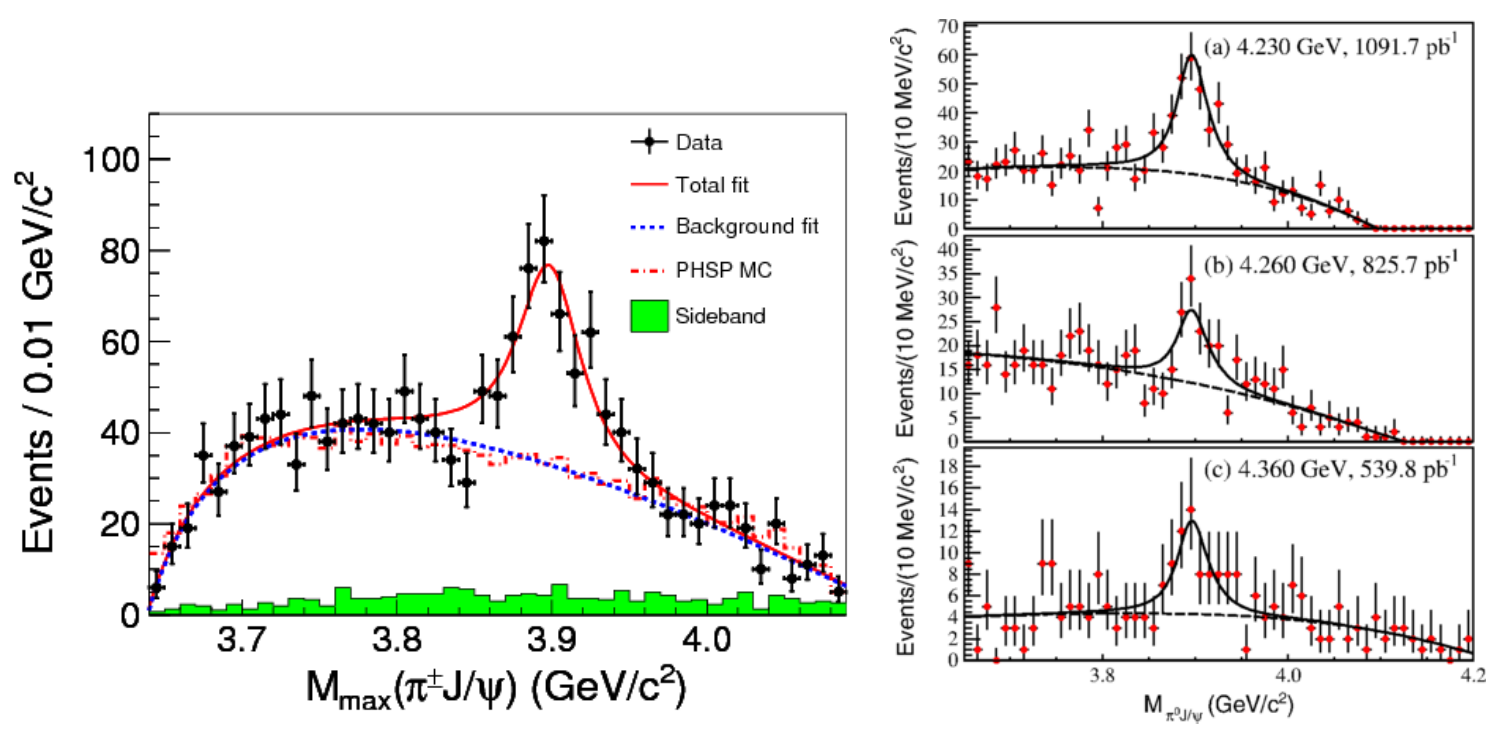

Figure 3: (Left) Fit to the invariant mass of $\pi^{ \pm} J / \psi$. (Right) A simultaneous fit to the $\pi^{0} J / \psi$ mass spectra at three different energy points.

using $525 \mathrm{pb}^{-1}$ data sample at $4.26 \mathrm{GeV}$. In the recoil mass of the bachelor pion, an enhancement was observed near the $D \bar{D}^{*}$ mass threshold, as shown in Fig. 4 (Left), named $Z_{c}(3885)$. The pole mass and width are determined to be $(3883.9 \pm 1.5 \pm 4.2) \mathrm{MeV} / \mathrm{c}^{2}$ and $(24.8 \pm 3.3 \pm 11.0)$ $\mathrm{MeV} / \mathrm{c}^{2}$, respectively. And the quantum number of $Z_{c}(3885)$ is studied by examining the polar angle distribution of the bachelor pion. The signal yield as a function of $\left|\cos \theta_{\pi}\right|$, shown in Fig. 4 (Right), agrees well with the flat expectation for $J^{P}=1^{+}[10]$.

Assuming the $Z_{c}(3885)$ structure is due to the $Z_{c}(3900)$, the ratio of partial decay widths is determined to be $\left[\left(\Gamma\left(Z_{c}(3885) \rightarrow D \bar{D}^{*}\right)\right) /\left(\Gamma\left(Z_{c}(3900) \rightarrow \pi J / \psi\right)\right)\right]=6.2 \pm 1.1 \pm 2.7$. This ratio is much smaller than the typical values for decays of the conventional charmonium states above the open charm threshold, which suggest the influence of very different dynamics in the $Y(4260)$ $Z_{c}(3900)$ system.
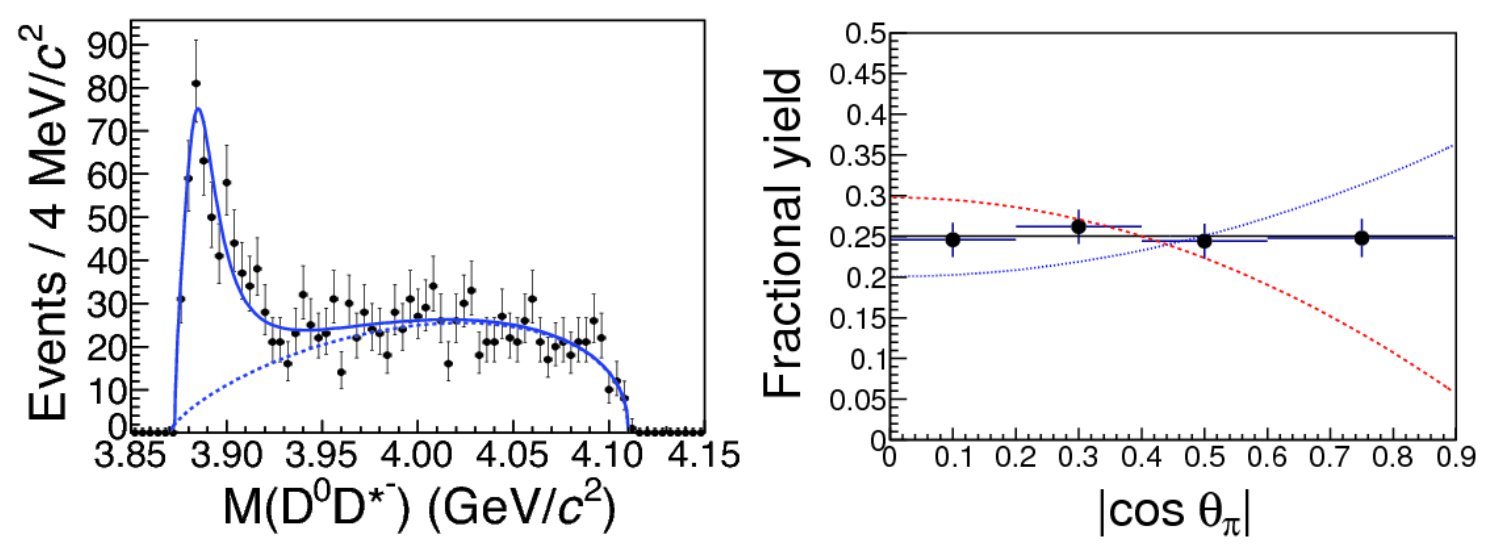

Figure 4: (Left) Fit to the invariant mass of $D^{0} D^{*-}$. (Right) Polar angle distribution of the bachelor pion, in which the solid, dashed, and dotted curves show expectations for $J^{P}=1^{+}, 0^{-}$, and $1^{-}$, respectively. 


\subsection{Observations of $Z_{c}(4020)$ and $Z_{c}(4025)$}

In order to search for other $Z_{c}$ states, studies on the process $e^{+} e^{-} \rightarrow \pi^{+} \pi^{-} h_{c}$ was performed at BESIII. In the $M\left(\pi^{ \pm} h_{c}\right)$ spectra, a structure around $4.02 \mathrm{GeV}$ is observed, as shown in Fig. 5 (Left), named as $Z_{c}(4020)$. The mass and width of this state are determined to be $(4022.9 \pm 0.8 \pm 2.7)$ $\mathrm{MeV} / \mathrm{c}^{2}$ and $(7.9 \pm 2.7 \pm 2.6) \mathrm{MeV} / \mathrm{c}^{2}$, respectively. The statistical significance is larger than 8.9 $\sigma[11]$.

Later its isospin partner was observed in the process $e^{+} e^{-} \rightarrow \pi^{0} \pi^{0} h_{c}$. In the $M\left(\pi^{0} h_{c}\right)$ spectra, an obvious peak around $4.02 \mathrm{GeV}$ is seen, as shown in Fig. 5 (Right). A fit on the mass spectra with width fixed to the value from charged channel is applied. The mass is determined to be (4023.6 \pm $2.2 \pm 3.9) \mathrm{MeV} / \mathrm{c}^{2}$, which is consistent with the charged $Z_{c}(4020)$. The statistical significance is larger than $5 \sigma$ [12].
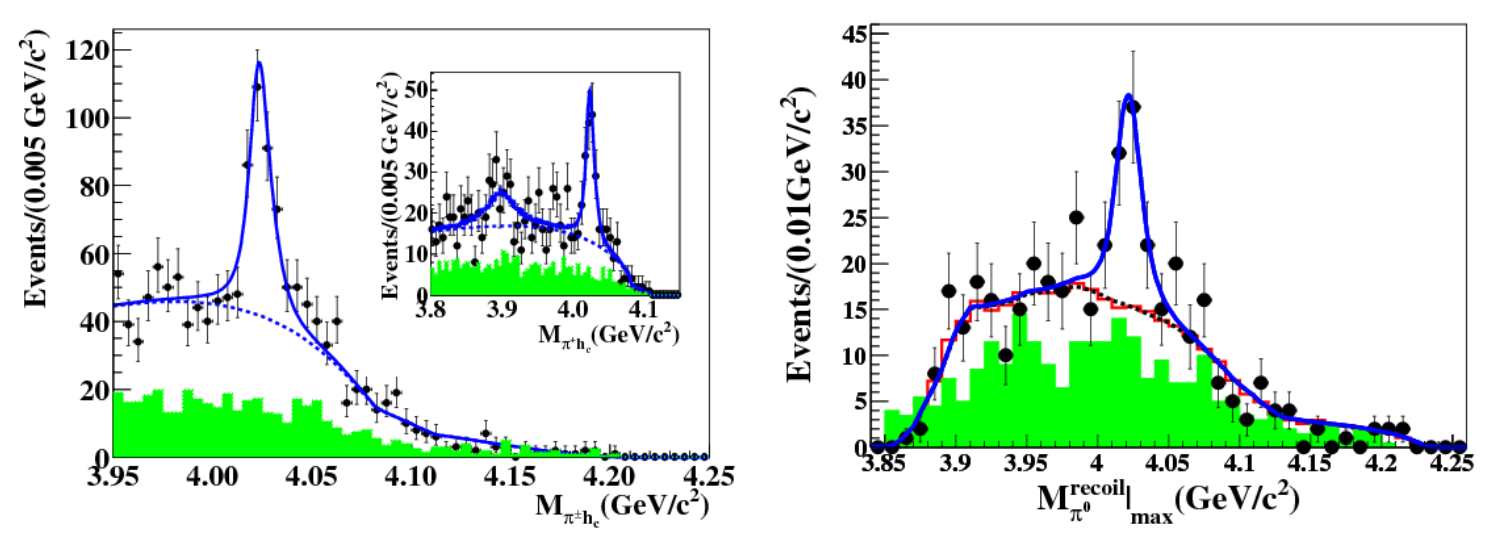

Figure 5: Fit to the invariant mass of $\pi^{ \pm} h_{c}$ (Left) and $\pi^{0} h_{c}$ (Right).

Since the mass of $Z_{c}(4020)$ is close to the $D^{*} \bar{D}^{*}$ threshold, a search of $Z_{c}$ states in the process of $e^{+} e^{-} \rightarrow \pi^{ \pm}\left(D^{*} \bar{D}^{*}\right)^{\mp}$ was performed. In the recoil mass of the bachelor pion, a structure near the $D^{*} \bar{D}^{*}$ threshold was observed as shown in Fig. 6 (Left), named as $Z_{c}(4025)$. The mass and width of this state are determined to be $(4026.3 \pm 2.6 \pm 2.7) \mathrm{MeV} / \mathrm{c}^{2}$ and $(24.8 \pm 5.6 \pm 7.7) \mathrm{MeV} / \mathrm{c}^{2}$, respectively [13].

Later its neutral partner, $Z_{c}(4025)^{0}$, was observed in the process $e^{+} e^{-} \rightarrow \pi^{0}\left(D^{*} \bar{D}^{*}\right)^{0}$, as shown in Fig. 6 (Right). Assuming a Breit-Wigner line shape, its pole mass and width are determined to be $\left(4025.5_{-4.7}^{+2.0} \pm 3.1\right) \mathrm{MeV} / \mathrm{c}^{2}$ and $(23.0 \pm 6.0 \pm 1.0) \mathrm{MeV} / \mathrm{c}^{2}$, respectively [14].

Assuming the $Z_{c}(4020)$ and $Z_{c}(4025)$ are the same state, the ratio of the partial decay widths is measured to be $\left[\left(\Gamma\left(Z_{c}(4025) \rightarrow D^{*} \bar{D}^{*}\right)\right) /\left(\Gamma\left(Z_{c}(4020) \rightarrow \pi h_{c}\right)\right)\right]=12 \pm 5$, which is also much smaller than that of the conventional charmonium states above open charm threshold.

\section{Summary}

Based on high luminosity data sets in BESIII, a relatively systematic study on exotic and charmonium-like states has been perform. Radiative and hadronic transitions between higher vector charmonium(-like) states to exotic/conventional charmonium states have also been investigated. Many interesting results have been obtained, which have greatly improved our knowledge about 

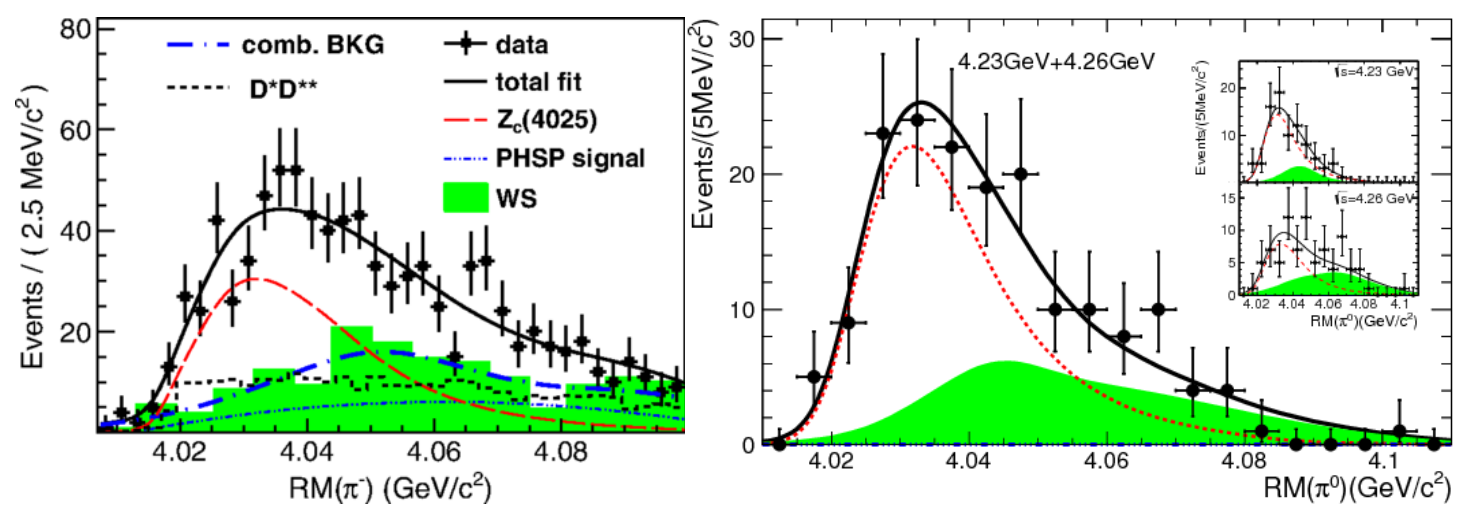

Figure 6: (Left) Fit to the recoil mass of $\pi^{-}$. (Right) Fit to the recoil mass of $\pi^{0}$

these exotic states. The observation of the charged $Z_{c}$ states may indicate one kind of the exotic states has been observed. However, it is still a big challenge to understand the nature of these exotic states. More data from BESIII experiment is expected to improve the current situation.

\section{References}

[1] H. Fritzsch, M. Gell-Mann and H. Leutwyler, Phys. Lett. B 47, 365 (1973)

[2] V. Bhardwaj et al. [Belle Collaboration], Phys. Rev. Lett. 111, 032001 (2013)

[3] M. Ablikim et al. [BESIII Collaboration], Phys. Rev. Lett. 115, 011803 (2015)

[4] S. K. Choi et al. [Belle Collaboration], Phys. Rev. Lett. 91, 262001 (2003)

[5] M. Ablikim et al. [BESIII Collaboration], Phys. Rev. Lett. 112, 092001 (2014)

[6] M. Ablikim et al. [BESIII Collaboration], Phys. Rev. Lett. 110, 252001 (2013)

[7] Z. Q. Liu et al. [Belle Collaboration], Phys. Rev. Lett. 110, 252002 (2013)

[8] T. Xiao, S. Bobbs, A. Tomaradze, Kamal K. Seth, Phys. Lett. B 727, 366 (2013)

[9] M. Ablikim et al. [BESIII Collaboration], Phys. Rev. Lett. 115, 112003 (2015)

[10] M. Ablikim et al. [BESIII Collaboration], Phys. Rev. Lett. 112, 022001 (2014)

[11] M. Ablikim et al. [BESIII Collaboration], Phys. Rev. Lett. 111, 242001 (2013)

[12] M. Ablikim et al. [BESIII Collaboration], Phys. Rev. Lett. 113, 212002 (2014)

[13] M. Ablikim et al. [BESIII Collaboration], Phys. Rev. Lett. 112, 132001 (2014)

[14] M. Ablikim et al. [BESIII Collaboration], arXiv: 1507.02404 